\title{
Quantum coherent plasmon in silver nanowires: A real-time TDDFT study
}

Feizhi Ding, ${ }^{1}$ Emilie B. Guidez, ${ }^{2}$ Christine M. Aikens, ${ }^{2, a)}$ and Xiaosong Li ${ }^{1}$,a)

${ }^{1}$ Department of Chemistry, University of Washington, Seattle, Washington 98195, USA

${ }^{2}$ Department of Chemistry, Kansas State University, Manhattan, Kansas 66506, USA

(Received 20 January 2014; accepted 6 June 2014; published online 25 June 2014)

\begin{abstract}
A plasmon-like phenomenon, arising from coinciding resonant excitations of different electronic characteristics in 1D silver nanowires, has been proposed based on theoretical linear absorption spectra. Such a molecular plasmon holds the potential for anisotropic nanoplasmonic applications. However, its dynamical nature remains unexplored. In this work, quantum dynamics of longitudinal and transverse excitations in 1D silver nanowires are carried out within the real-time time-dependent density functional theory framework. The anisotropic electron dynamics confirm that the transverse transitions of different electronic characteristics are collective in nature and oscillate in-phase with respect to each other. Analysis of the time evolutions of participating one-electron wave functions suggests that the transverse transitions form a coherent wave packet that gives rise to a strong plasmon resonance at the molecular level. (C) 2014 AIP Publishing LLC. [http://dx.doi.org/10.1063/1.4884388]
\end{abstract}

\section{INTRODUCTION}

When a gold or silver nanoparticle is subjected to an electromagnetic field with a photon frequency corresponding to a natural frequency of the valence electrons collectively oscillating against the restoring force of the positive nuclei, a strong peak appears in the absorption spectrum. ${ }^{1-3}$ This collective oscillation leads to an enhanced and confined electric field around the particle. ${ }^{3-5}$ This phenomenon, called surface plasmon resonance (SPR), has been intensively studied both experimentally ${ }^{6-10}$ and theoretically. ${ }^{11-16}$ Applications include high-sensitivity chemical and biological sensing ${ }^{17-25}$ as well as energy conversion and storage. ${ }^{26,27}$ Anisotropic systems such as nanorods and nanowires are especially useful for certain applications such as photothermal cancer therapy. ${ }^{28,29}$

Noble metal nanorods have two main plasmon modes: the longitudinal mode (along the main axis of the nanorod) and the transverse mode (in the plane perpendicular to the main axis). ${ }^{22,30-34}$ The longitudinal peak observed in the excitation spectrum shifts to lower energy with increasing aspect ratio whereas the transverse peak remains at nearly constant energy. ${ }^{30-34}$ Recent linear response time-dependent density functional theory (LR-TDDFT) calculations performed on noble metal clusters suggest that a plasmon may be identified when there is a constructive addition of the dipole moments of one-electron transitions and does not only occur in large nanoparticles (from a few to several hundred nanometers) but also in small clusters (less than $2 \mathrm{~nm}$ ). ${ }^{34-40}$ The frontier orbitals of silver and gold clusters are linear combinations of the valence s-orbitals of the metal atoms and are delocalized over the entire structure. ${ }^{12,37,41}$

Linear chains of noble metal atoms (nanowires) have been studied as model systems using TDDFT..$^{37,39,42-45}$ While

\footnotetext{
a) Authors to whom correspondence should be addressed. Electronic
} addresses: cmaikens@ksu.edu and li@chem.washington.edu the frequency domain LR-TDDFT method has been routinely used for studying plasmon excitations in small metal clusters, it does not offer insights into the dynamical nature of the interplay between electronic excited states. On the other hand, the real-time (RT) TDDFT method has emerged as a powerful tool to provide a straightforward, meticulous elucidation on the time evolution of the system subject to any external perturbation. For example, using real-time TDDFT, Yan et al. ${ }^{43,44}$ identified the end and central plasmon resonances in linear sodium and silver chains. Gao et al. ${ }^{45}$ studied the formation and size-dependence of electronic excitations in copper, silver, and gold chains with up to 26 atoms. In both studies, the collective nature of the longitudinal and transverse excitations was deduced by the length dependence of the dipole strength. Induced density response was also analyzed to understand the nature of the two resonance modes.

Due to the symmetry of the nanowires, the delocalized frontier orbitals have cylindrical symmetry and can be labeled with the Greek letters $\Sigma, \Pi, \Delta$, etc. The longitudinal peak of these systems corresponds to the HOMO $\rightarrow$ LUMO transition $(\Sigma \rightarrow \Sigma)$. The transverse peak corresponds to a superposition of $\Sigma \rightarrow \Pi$ transitions whose dipole moments add up constructively (a plasmon according to the identification scheme mentioned above) ${ }^{37}$ However, the dynamical nature of such a superposition of single-particle transitions (i.e., molecular orbital transitions) is still unknown. How this superposition affects the time-dependence of the electron density in the plasmonic excitation remains unexplored.

To this end, we use the real-time TDDFT method developed in our group to study the interplay between the time evolution of the superposition of the single-particle transitions and the plasmonic excitations in silver chains. Formation and length-dependence of the longitudinal and transverse excitations are investigated by probing the dipole response to an external step function electric field. In particular, we explore the superposition nature of the transverse excitation by analyzing the time evolution of the molecular orbital occupations. We 
observe a collective oscillation of single-particle transitions that are in-phase and coherent. This phenomenon is linked to the collective nature of plasmon resonance.

The paper is organized as follows: in Sec. II we describe the implementation of the real-time TDDFT method and computational details, followed by results and discussion in Sec. III. Concluding remarks are given in Sec. IV.

\section{METHODOLOGY}

The plasmon excitations of the silver nanowires are examined by performing the RT-TDDFT calculations, in which the electronic density matrix is propagated according to the TDDFT equation:

$$
i \frac{d \mathbf{P}(t)}{d t}=[\mathbf{K}(t), \mathbf{P}(t)],
$$

where $\mathbf{P}$ and $\mathbf{K}$ are density and Kohn-Sham matrices in orthonormal basis. Atomic units (a.u.) are used throughout this paper. Equation (1) is integrated with a modified midpoint and unitary transformation (MMUT) algorithm, ${ }^{46,47}$ where the density matrix is propagated using a unitary time evolution operator $\mathbf{U}\left(t_{n}\right)$ :

$$
\mathbf{P}\left(t_{n+1}\right)=\mathbf{U}\left(t_{n}\right) \cdot \mathbf{P}\left(t_{n-1}\right) \cdot \mathbf{U}^{\dagger}\left(t_{n}\right),
$$

where $\mathbf{U}\left(t_{n}\right)$ is constructed from the eigenvectors $\mathbf{C}\left(t_{n}\right)$ and eigenvalues $\boldsymbol{\epsilon}\left(t_{n}\right)$ of the Kohn-Sham matrix at time $t_{n}$ :

$$
\begin{aligned}
\mathbf{U}\left(t_{n}\right) & =\exp \left[-i \cdot 2 \Delta t \cdot \mathbf{K}\left(t_{n}\right)\right] \\
& =\mathbf{C}\left(t_{n}\right) \cdot \exp \left[-i \cdot 2 \Delta t \cdot \boldsymbol{\epsilon}\left(t_{n}\right)\right] \cdot \mathbf{C}^{\dagger}\left(t_{n}\right)
\end{aligned}
$$

with $\Delta t$ denoting the time step.

To probe the time-dependent response of the silver chains to external electric field perturbations, we apply a stepfunction electric field either along the longitudinal or along the transverse direction:

$$
\mathbf{E}(t)=\left\{\begin{array}{ll}
\mathbf{E}_{0} & t<0 \\
0 & t>0
\end{array} .\right.
$$

Computationally this is equivalent to the following scheme: (1) prepare the initial electronic density by a self-consistentfield (SCF) calculation in the presence of the static electric field $\mathbf{E}_{0}$; (2) turn off the field and propagate the electronic system using the RT-TDDFT method outlined above.

The time-dependent dipole moment $\boldsymbol{\mu}(t)$ is calculated at each time step according to

$$
\boldsymbol{\mu}(t)=\operatorname{Tr}[\mathbf{D P}(t)],
$$

where $\mathbf{D}$ is the dipole matrix in the orthonormal basis. With the dipole response in real time, one can compute the dipole strength function $S(\omega)$, which is given by

$$
S(\omega)=\frac{4 \pi \omega}{3 c} \operatorname{Tr}[\operatorname{Im} \boldsymbol{\alpha}(\omega)],
$$

where $\boldsymbol{\alpha}(\omega)$ is the polarizability in the frequency domain and it can be obtained by the Fourier transform of the dipole moment and the step field through the following relation:

$$
\mu_{i}(\omega)=\alpha_{i i}(\omega) E_{i}(\omega),
$$

where $i$ stands for $x, y, z$ Cartesian coordinates.
At the weak perturbation limit, the time-domain approach generates the same excitation energies and relative dipole strengths as the frequency-domain LR-TDDFT. ${ }^{48}$ On the other hand, the time-domain method can reveal insightful dynamical information about the interplay between the system and the external field, as will be discussed in Sec. III.

To find the relationship between the transverse excitation and single-particle transitions, we analyze the time evolutions of the molecular orbital occupations, which are calculated by the projection of the time-dependent density matrix onto the initial ground state orbital space: ${ }^{46}$

$$
n_{i}\left(t_{k}\right)=\mathbf{C}_{i}^{\dagger}\left(t_{0}\right) \mathbf{P}\left(t_{k}\right) \mathbf{C}_{i}\left(t_{0}\right),
$$

where $n_{i}$ is the occupation of the $i$ th molecular orbital.

\section{RESULTS AND DISCUSSION}

The RT-TDDFT simulations were carried out using the development version of the GAUSSIAN series of programs. ${ }^{49}$ A series of linear silver chains, with 4, 6, 8, 10, and 12 atoms were investigated. In all calculations, the BP86 density functional and the LanL2DZ ${ }^{50-52}$ effective core potential were used. For real time simulations, the electronic wavepacket was evolved for 100 fs with an integration step size of 0.5 a.u. ( $\sim 1.2$ as). The strength of the external static field was chosen to be 0.001 a.u. In doing the Fourier transform of the time-dependent dipole moments, we added a damping factor of $0.2 \mathrm{eV}$ which can physically account for the experimental broadening of the dipole strength. The molecular geometries of the studied silver linear chains were taken from Ref. 37.

The dipole responses of the silver chains due to an external step-function field perturbation along the longitudinal and transverse directions are shown in Fig. 1. Two main peaks, arising from longitudinal and transverse perturbations, respectively, are observed within the energy range of $0-8 \mathrm{eV}$ for all chains. This is consistent with the linear response TDDFT calculations in Ref. 37. The longitudinal peak is redshifted when the chain length increases whereas the frequency of the transverse peak remains relatively constant, only slightly blueshifting, as seen in Fig. 2(a). Similar trends were also observed in Refs. 44 and 45. Figure 2(b) plots the dipole strength as a function of the length of the silver nanowire. The dipole strength of both the longitudinal and transverse excitations increases as the chain length increases.

As shown in linear response TDDFT calculations, ${ }^{37}$ the longitudinal excitation is dominated by a single-particle transition (e.g., HOMO $\rightarrow$ LUMO transition), which was assigned as a plasmon excitation. ${ }^{37,44,45}$ In classical solid-state physics, there is a clear distinction between single-particle excitations and plasmon excitations. ${ }^{39}$ However, for systems consisting of a few to hundreds of atoms, classical electrodynamics is no longer applicable due to the existence of discrete energy levels in small clusters, and the distinction between single-particle excitations and plasmon excitations becomes less obvious. Identification of plasmon excitations in cases where the excitation is dominated by a single-particle transition, such as that in the longitudinal excitation, is not well-established in the field, but an identification procedure has recently been proposed that demonstrates the plasmonic 

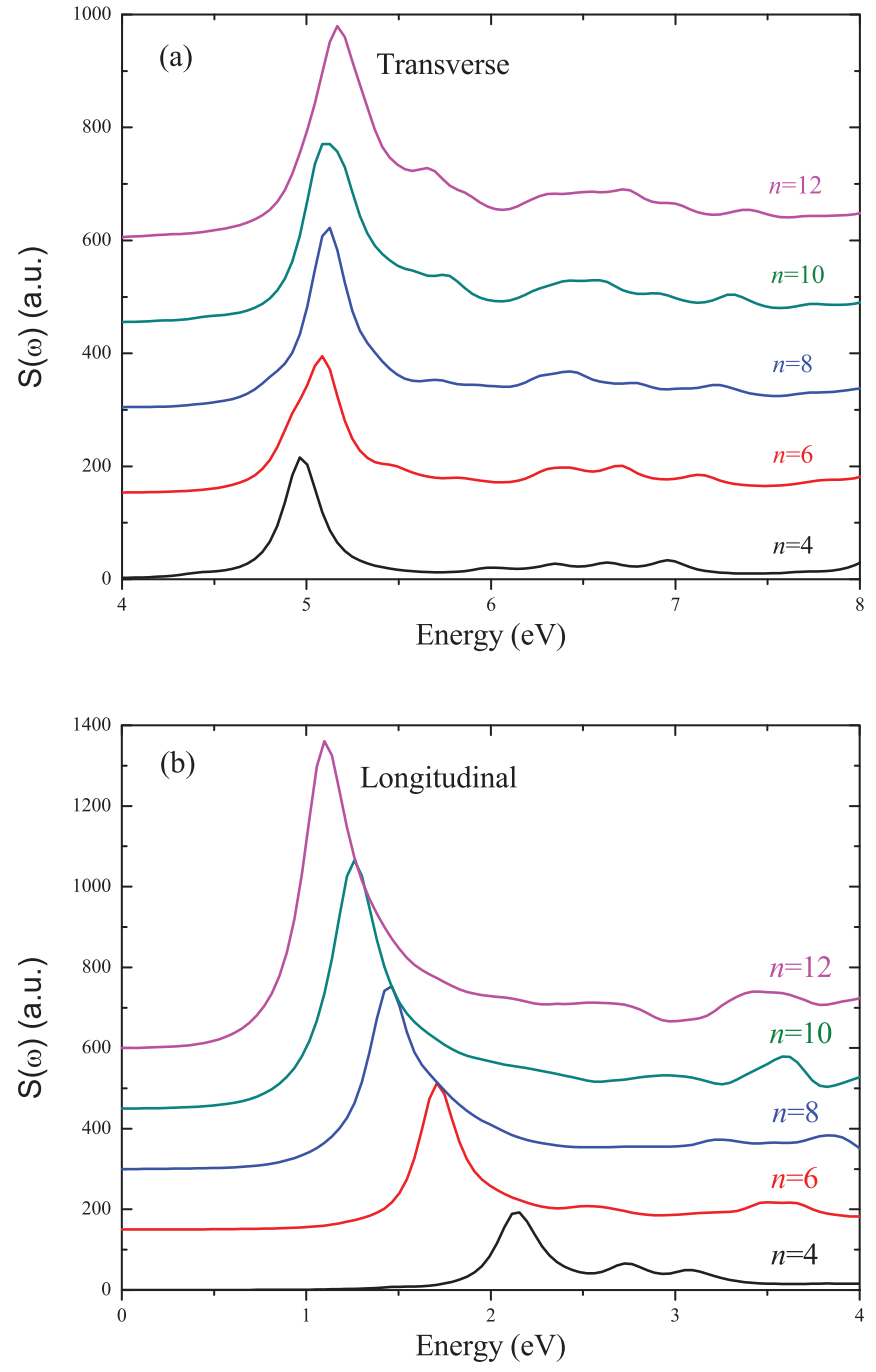

FIG. 1. Fourier spectra of dipole response of linear silver chains $\operatorname{Ag}_{n}(n=4$, $6,8,10,12$ ), perturbed by an external step field polarized in the (a) longitudinal and (b) transverse directions. Different spectra are shifted upward by 150 a.u. for clarity.

character of the longitudinal transition. ${ }^{39}$ The transverse excitation, on the other hand, can be characterized by a superposition of multiple single-particle transitions, e.g., $\Sigma_{m} \rightarrow \Pi_{m}$ for $\mathrm{Ag}_{2 m}$, as indicated by linear response calculations. ${ }^{37}$ For the $\mathrm{Ag}_{6}$ nanowire (Table I), one transverse peak $\left(\mathrm{T}_{1}\right)$ with strong oscillator strength arises due to constructive interaction between the $\Sigma_{1} \rightarrow \Pi_{1}, \Sigma_{2} \rightarrow \Pi_{2}$, and $\Sigma_{3} \rightarrow \Pi_{3}$ single-particle transitions, whereas two transverse peaks $\left(\mathrm{T}_{2}\right.$ and $\left.\mathrm{T}_{3}\right)$ arise from the destructive interaction of these three transitions. As recently shown by Guidez and Aikens, ${ }^{53}$ a simple configuration interaction (CI) picture in which three Koopman-like energy differences $\left(\alpha_{1}=E\left(\Pi_{1}\right)-E\left(\Sigma_{1}\right), \alpha_{2}=E\left(\Pi_{2}\right)-E\left(\Sigma_{2}\right)\right.$, and $\left.\alpha_{3}=E\left(\Pi_{3}\right)-E\left(\Sigma_{3}\right)\right)$ are coupled by off-diagonal elements $\beta_{I J}$ representing the coupling between the singly excited configurations $\Phi_{I}$ and $\Phi_{J}$ given by

$$
\beta_{I J}=\left\langle\Phi_{I}|\hat{H}| \Phi_{J}\right\rangle
$$

can be employed to understand the constructive and destructive combinations. At the LB94/DZ level of theory, the val-
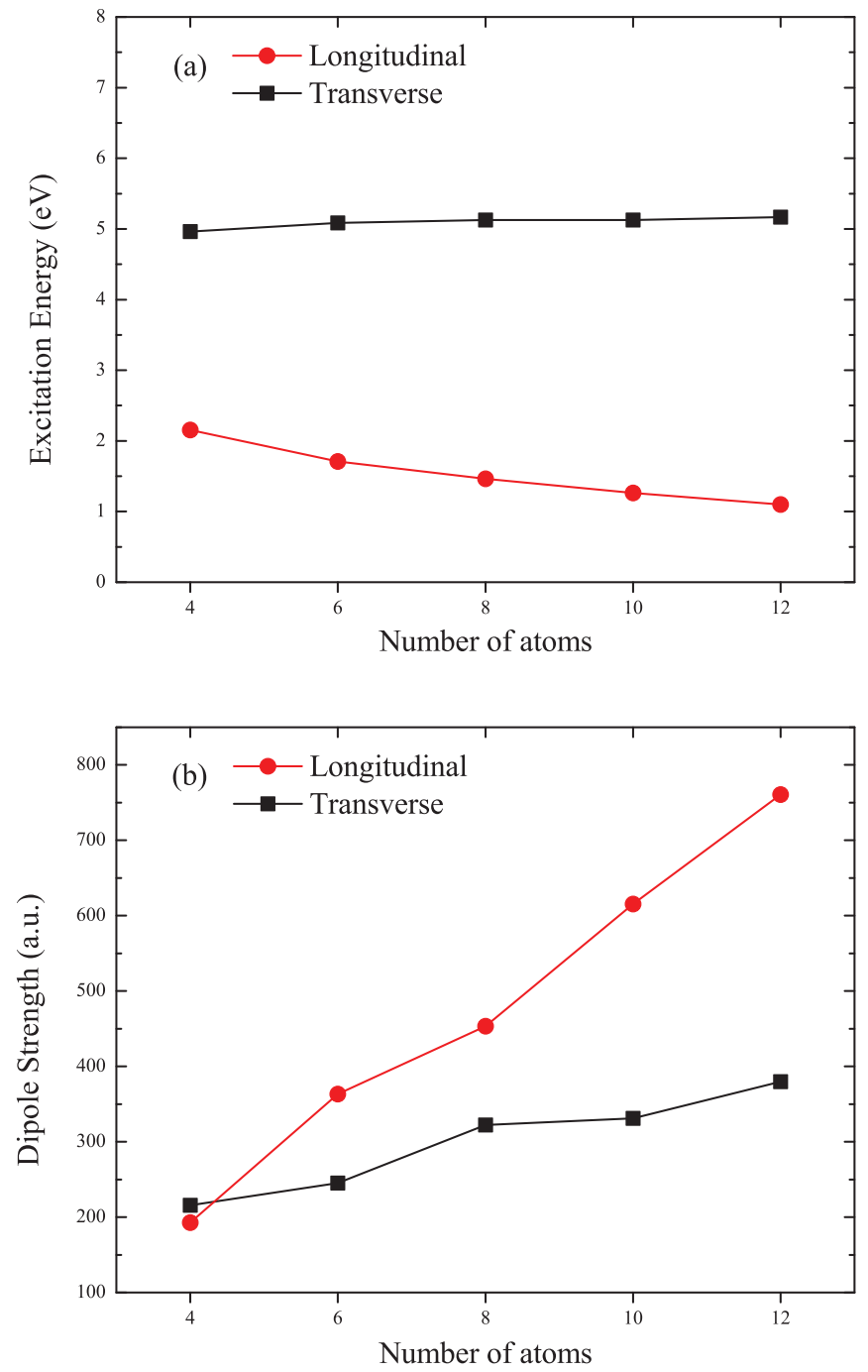

FIG. 2. (a) Excitation energy and (b) dipole strength as functions of the length (number of atoms) of silver nanowire for the longitudinal and transverse plasmon resonant peaks shown in Fig. 1.

ues of $\alpha$ obtained from a Koopman-like approximation are $\alpha_{1}=5.59 \mathrm{eV}, \alpha_{2}=5.69 \mathrm{eV}$, and $\alpha_{3}=5.79 \mathrm{eV}$. Coupling of the three single-particle transitions leads to one peak at higher energy with strong oscillator strength and two peaks at lower energy with near-zero oscillator strength, as observed in Table I. In this CI picture, essentially all of the oscillator strength that would normally belong to individual singleparticle transitions is present in a single collective peak. One interpretation of the $\mathrm{T}_{1}$ peak shown in Table $\mathrm{I}$ is that it corresponds to a collective excitation of all of the conduction band electrons (i.e., for $\mathrm{Ag}_{6}$, those in $\Sigma_{1}, \Sigma_{2}$, and $\Sigma_{3}$ ), which is one definition of a plasmon. In this work, we place special emphasis on the dynamical nature of this collective transverse excitation.

To probe the nature of the interplay between these singleparticle transitions, the time evolution of the electron density is analyzed by projecting to the ground-state molecular orbital space using Eq. (8), resulting in time-dependent occupation numbers of pertinent orbitals. The phase relationship between the time evolutions of the orbital occupation numbers will provide information regarding the constructive or 
TABLE I. Excitation energies, oscillator strengths, single-particle transitions, and dipole moment contribution arising from each single-particle transition, for the longitudinal (L) and transverse ( $\mathrm{T}$ ) peaks of the $\mathrm{Ag}_{6}$ nanowire at the LB94/DZ level of theory (Ref. 37).

\begin{tabular}{lcccr}
\hline \hline Peak & $\begin{array}{c}\text { Energy } \\
(\mathrm{eV})\end{array}$ & $\begin{array}{c}\text { Oscillator } \\
\text { strength (a.u.) }\end{array}$ & Transitions & $\begin{array}{c}\text { Dipole moment } \\
\text { contributions (a.u.) }\end{array}$ \\
\hline $\mathrm{L}$ & 1.98 & 1.74 & $\Sigma_{3} \rightarrow \Sigma_{4}$ & -6.3581 \\
& & & $\Sigma_{1} \rightarrow \Pi_{1}$ & 0.6997 \\
$\mathrm{~T}_{1}$ & 6.86 & 0.67 & $\Sigma_{2} \rightarrow \Pi_{2}$ & 1.0356 \\
& & & $\Sigma_{3} \rightarrow \Pi_{3}$ & 0.9631 \\
& & & $\Sigma_{1} \rightarrow \Pi_{1}$ & 0.3590 \\
$\mathrm{~T}_{2}$ & 5.75 & 0.0011 & $\Sigma_{2} \rightarrow \Pi_{2}$ & 1.5259 \\
& & & $\Sigma_{3} \rightarrow \Pi_{3}$ & -1.8127 \\
& & & $\Sigma_{1} \rightarrow \Pi_{1}$ & -1.9817 \\
$\mathrm{~T}_{3}$ & 5.82 & 0.0013 & $\Sigma_{2} \rightarrow \Pi_{2}$ & 1.1369 \\
& & & $\Sigma_{3} \rightarrow \Pi_{3}$ & 0.6165 \\
\hline \hline
\end{tabular}

destructive nature of related electronic transitions. In addition, if the excited state wave functions are quantum mechanically coherent, the non-zero off-diagonal probability density $\left(\rho_{i j} \cdot e^{-i\left(\omega_{j}-\omega_{i}\right) t}\right)$ will give rise to an additional peak in the spectrum of population density at a frequency that corre-
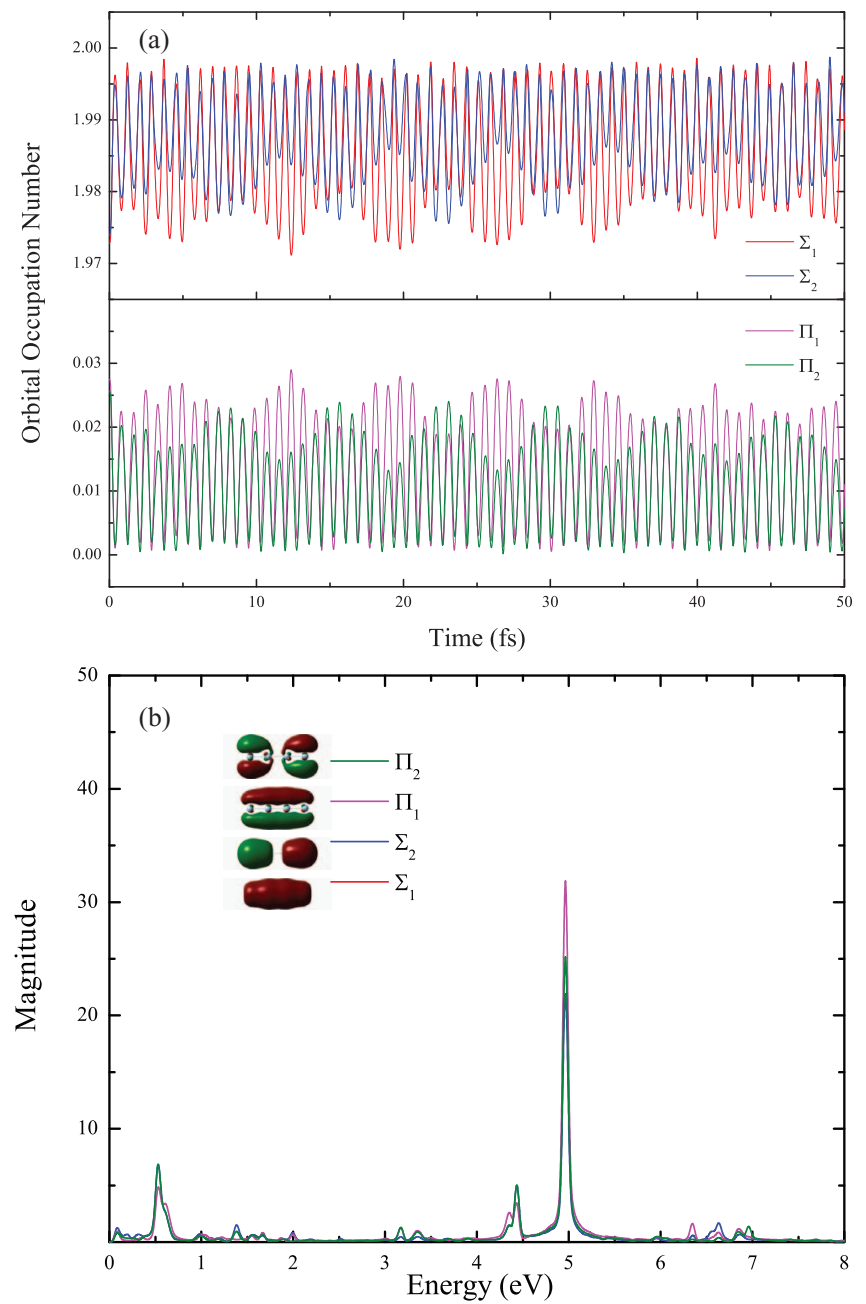

FIG. 3. Orbital occupation analysis for the $\mathrm{Ag}_{4}$ chain: (a) time-dependent oscillations and (b) the corresponding Fourier transformed spectra. sponds to the frequency difference $\left(\omega_{j}-\omega_{i}\right)$ of participating states. As a result, any component (e.g., participating $\mathrm{MO}$ occupation number) of the state population density also bears the same signature of coherence. Therefore, a frequency analysis of the time evolution of orbital occupation numbers can be used to probe the coherence of electronic excited states. Although the overall electronic density is evolved in a closed system formalism with no decoherence, the projected subsystem of interest (i.e., plasmon) is allowed to interact with other empty orbitals that may result in the resonant dephasing mechanism and a phase shift in orbital occupation oscillation if it does exist in the simulations. In other words, microscopic dephasing mechanism is allowed from the sub-system point of view even though the total system is energetically closed.

Figure 3 shows the time evolution of the occupation numbers of the four single-particle orbitals $\left(\Sigma_{1}, \Sigma_{2}, \Pi_{1}\right.$, and $\Pi_{2}$ ), which are involved in the transverse excitation in the $\mathrm{Ag}_{4}$ nanowire. For $\mathrm{Ag}_{4}$, the transverse excitation consists of two single-particle transitions: $\Sigma_{1} \rightarrow \Pi_{1}$ and $\Sigma_{2} \rightarrow \Pi_{2}$. Fourier transforms of these oscillations resolved a strong peak at $\sim 5 \mathrm{eV}$ (Fig. 3(b)), agreeing with the transverse peak in the total dipole strength spectrum in Fig. 1. This observation suggests that these single-particle excitations are indeed responsible for the main absorption peak in Fig. 1. As these
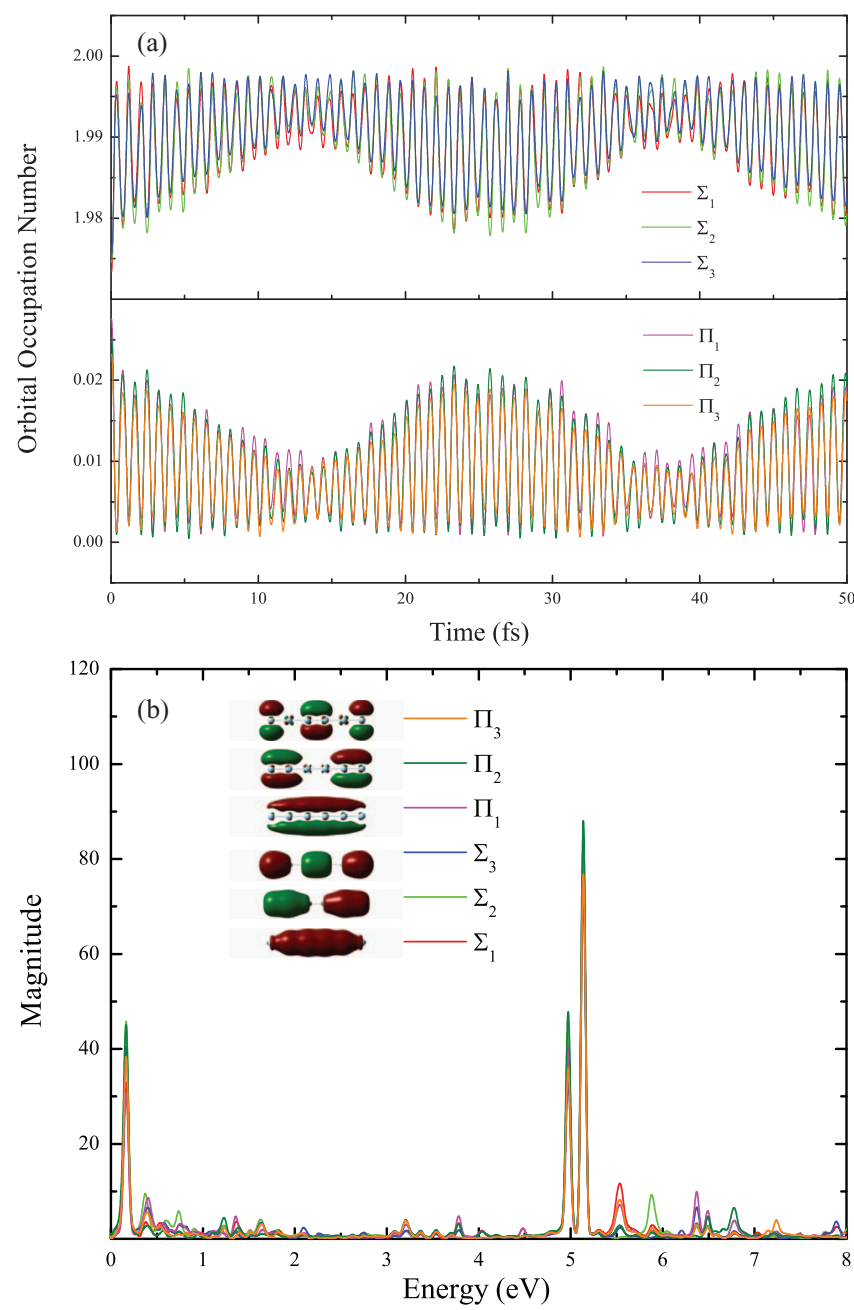

FIG. 4. Orbital occupation analysis for the $\mathrm{Ag}_{6}$ chain: (a) time-dependent oscillations and (b) the corresponding Fourier transformed spectra. 

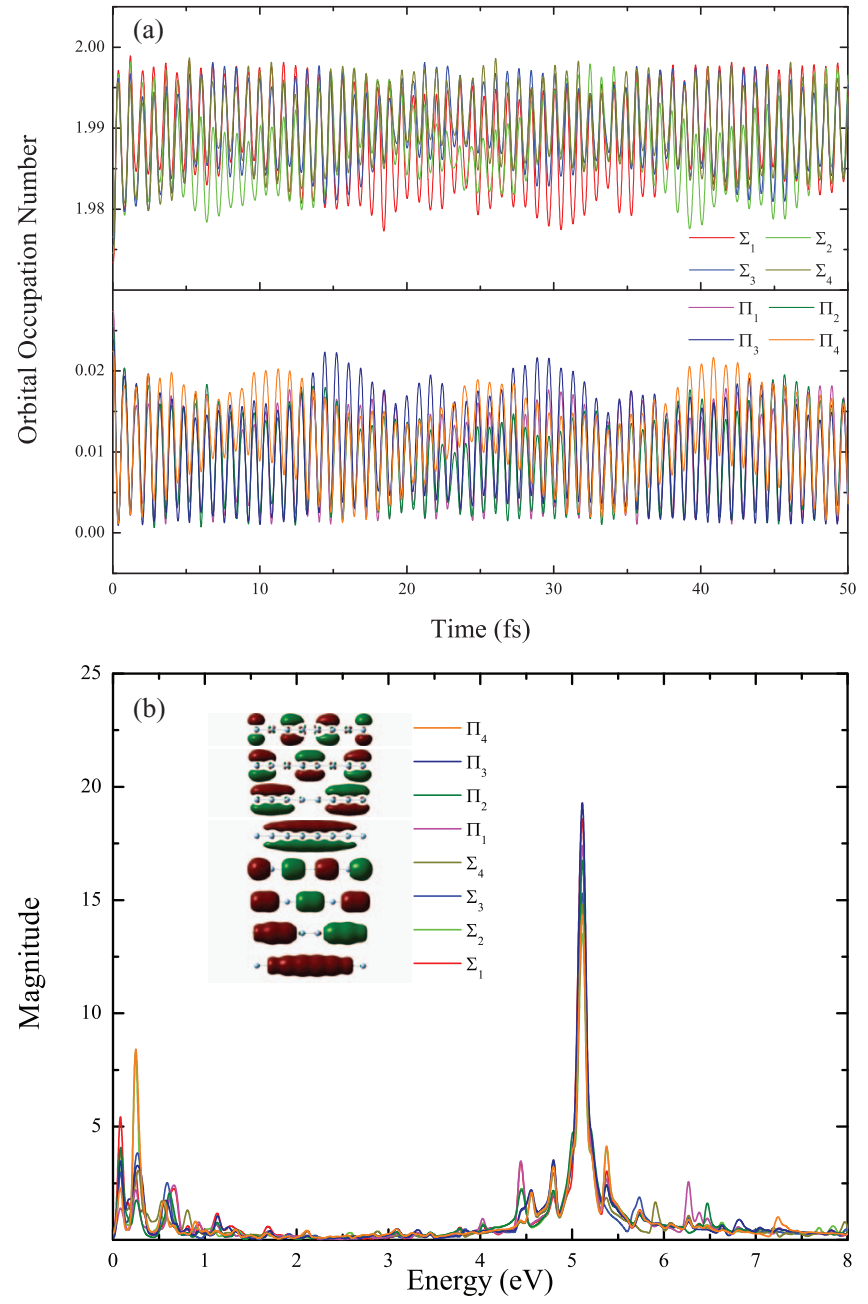

FIG. 5. Orbital occupation analysis for the $\mathrm{Ag}_{8}$ chain: (a) time-dependent oscillations and (b) the corresponding Fourier transformed spectra.

transitions occur at a similar frequency, they can form the so-called "excited state plasmon" 54 if the interactive nature of these different electronic transitions is constructive. As shown in Fig. 3(a), the oscillations of the four orbital occupations are in phase and constructively interfere with each other. The occupation numbers of $\Sigma_{1}\left(\Sigma_{2}\right)$ and $\Pi_{1}\left(\Pi_{2}\right)$ oscillate with the same amplitude, indicating a pairwise transition between $\Sigma_{1}\left(\Sigma_{2}\right)$ and $\Pi_{1}\left(\Pi_{2}\right)$. The phase-relationship of these oscillations is well-maintained throughout simulations of much longer time scale (longer time simulation, $>100 \mathrm{fs}$, is not shown here). This implies that the transverse excitation is composed of constructive and collective oscillations of two single-particle transitions with no interference from other excited states. Although the two participating single-particle transitions are of very different electronic characteristics, the collective nature of the overall transverse excitation gives rise to a plasmon-like character. A peak appears at $\sim 0.5 \mathrm{eV}$ in the spectrum of the time evolution of population density, which does not show up in the dipole strength spectrum. The frequency of this peak $(\sim 0.5 \mathrm{eV})$ is equal to the frequency difference between the two peaks at 4.5 and $5 \mathrm{eV}$. As discussed previously, this is an indication of the quantum coherence of the two single-particle transitions, $\Sigma_{1}$ $\rightarrow \Pi_{1}$ and $\Sigma_{2} \rightarrow \Pi_{2}$. Such a quantum coherence gives an ad-
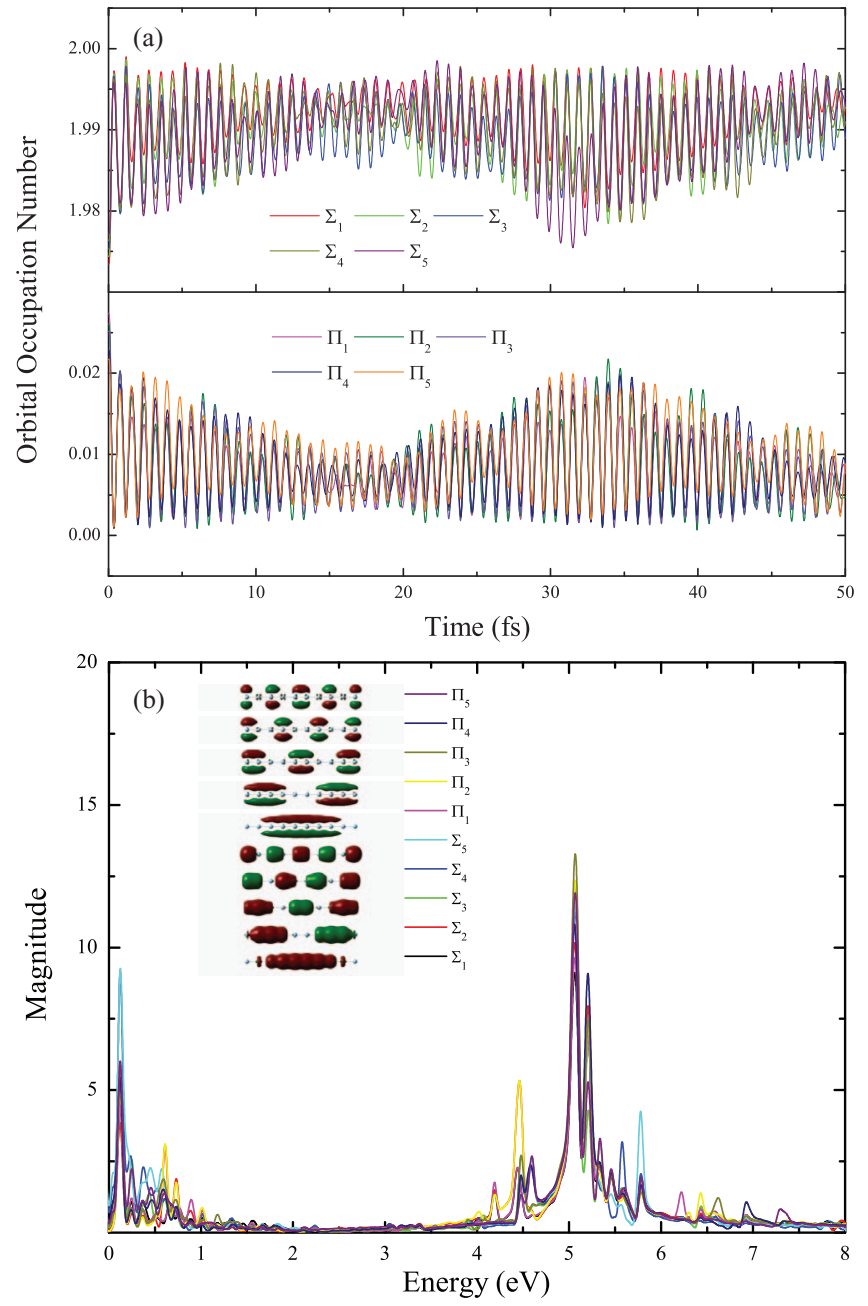

FIG. 6. Orbital occupation analysis for the $\mathrm{Ag}_{10}$ chain: (a) time-dependent oscillations and (b) the corresponding Fourier transformed spectra.

ditional characteristics to the "excited state plasmon" in systems with quantized energy levels.

For $\mathrm{Ag}_{6}$, the transverse peak consists of three singleparticle transitions: $\Sigma_{1} \rightarrow \Pi_{1}, \Sigma_{2} \rightarrow \Pi_{2}$, and $\Sigma_{3} \rightarrow \Pi_{3}$. Figure 4 shows the occupation number time evolutions of the six orbitals and the corresponding Fourier transform spectra. The oscillations are in phase and constructively interfere with each other, and the phase relationship is again well maintained throughout the simulation. Slightly different from $\mathrm{Ag}_{4}$, all six orbitals oscillate with comparable amplitude. Pairwise transitions may still be identified from the character of the molecular orbitals, as depicted in Fig. 4. Fourier transforms of the six oscillations result in a similar spectrum. The two strong peaks at $\sim 5 \mathrm{eV}$ correspond to the broadened transverse peak in the dipole strength spectrum, confirming that the transverse excitation is a collective oscillation of three single-particle transitions. The appearance of an additional peak at $\sim 0.2 \mathrm{eV}$ results from the frequency difference between the two closely spaced peaks at $\sim 5 \mathrm{eV}$, indicating a quantum coherence nature of the three single-particle transitions. These analyses again suggest that the in-phase and quantum coherent single-particle transitions in the transverse direction can lead to an excited state plasmon at the molecular level. 

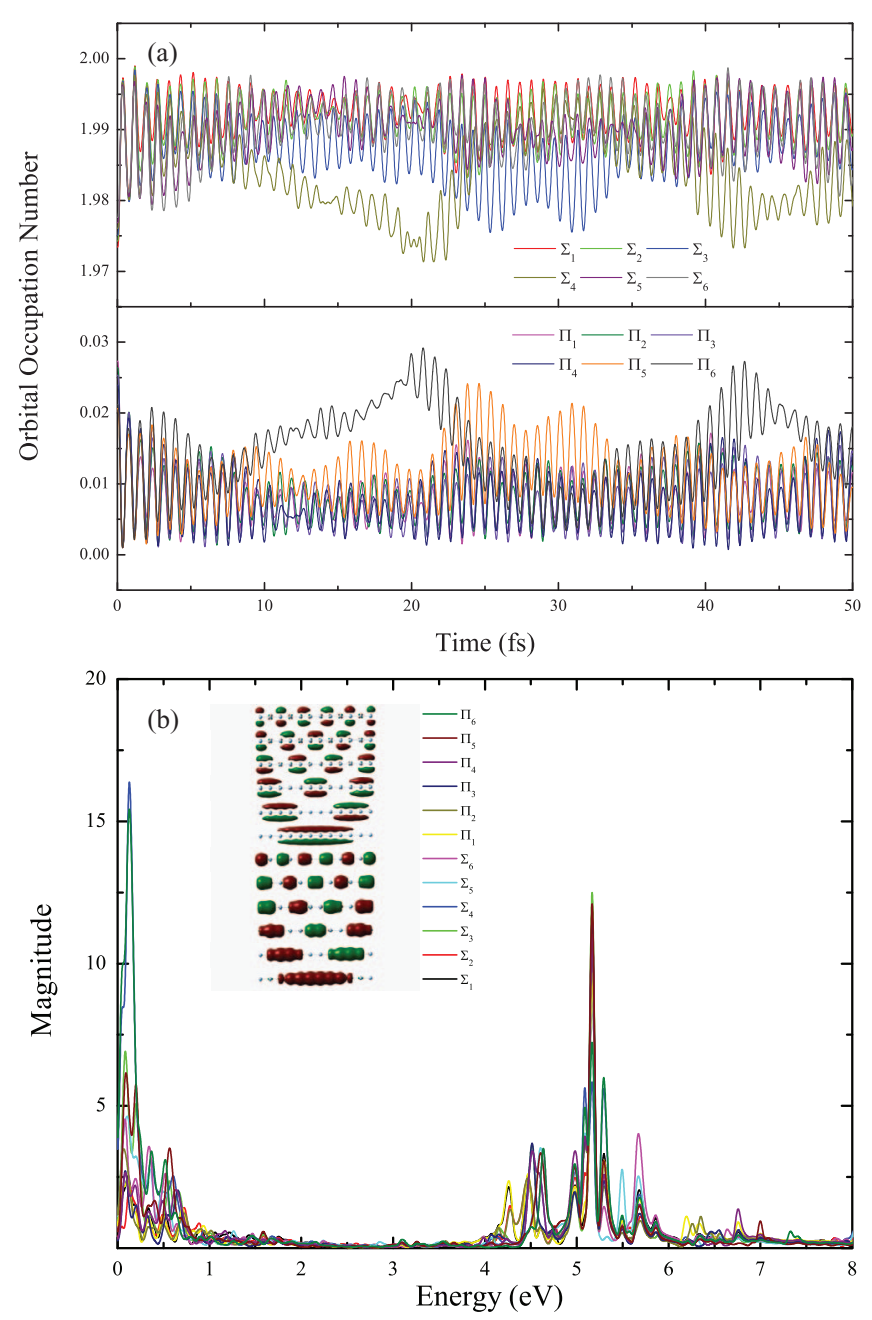

FIG. 7. Orbital occupation analysis for the $\mathrm{Ag}_{12}$ chain: (a) time-dependent oscillations and (b) the corresponding Fourier transformed spectra.

Starting at $\mathrm{Ag}_{8}, d$-transitions start to mix with the $\Sigma$ $\rightarrow \Pi$ transitions for the transverse excitation. ${ }^{37}$ Although the single-particle transitions $\Sigma \rightarrow \Pi$ still make major contributions to the transverse excitation, the participation of the $d$-transitions will certainly complicate the analysis. Broad absorption peaks will show up in the transverse spectra because of the mixed $d$-transitions, which was also observed previously. ${ }^{44}$ In Fig. 5 we plot the orbital occupation number oscillations involved in the four single-particle transitions in $\mathrm{Ag}_{8}$. These oscillations are of similar frequency and constructively interfere with each other, confirming again the collective nature of this excitation. Compared to $\mathrm{Ag}_{4}$ and $\mathrm{Ag}_{6}$, the $\mathrm{Ag}_{8}$ chain exhibits a larger variation in the amplitude of orbital oscillations and identification of the pairwise transitions becomes less trivial. Fourier transforms of the orbital oscillations show a broadened peak at $\sim 5 \mathrm{eV}$ with a complex pattern in the low frequency regime, as a result of the quantum coherence of all four single-particle transitions. For longer chains such as $\mathrm{Ag}_{10}$ and $\mathrm{Ag}_{12}$, although the dynamical interplays between participating single-particle transitions are even more complex, we still can observe oscillations of the single-particle transitions that are in-phase and constructively interfere with each other, and low frequency peaks resulting from quantum coherence (Figs. 6 and 7).

\section{CONCLUSION}

Using the real-time TDDFT method, we have studied the electronic excitations in a series of 1D silver nanowires. Length-dependence of the excitation energies and dipole strengths is investigated by analyzing the dipole response of the system to an external step function field in the longitudinal and transverse directions. We placed a special emphasis on the uniqueness of the transverse transitions from the point of view of dynamics. The time evolution of the population density of orbitals associated with the transverse excitation has been analyzed. In-phase and constructive oscillations of the associated single-particle transitions are observed, creating the plasmon. What makes this molecular plasmon unique is the quantum coherence of the single-particle transitions as suggested by the low frequency peaks in Fourier transforms of time-evolutions of orbital occupation numbers. It is this strong quantum coherence among these characteristic electronic oscillations that gives rise to molecular-scale plasmonics and nanoplasmonics, and our work herein provides the theoretical evidence. We also believe this feature could also exist in many quantum confined systems, such as quantum confined semiconductor nanocrystals and conjugated polymers. In this work, we simulate an energetically closed system with no decoherence or interaction with external baths. The goal of this work is to illustrate the existence of quantum coherent plasmon. Plasmonic de-phasing and decoherence will be addressed in future work.

\section{ACKNOWLEDGMENTS}

This material is based on work supported by the U.S. National Science Foundation (CHE-0844999 and CHE-1265945 to X.L., and EPS-0903806 to C.M.A.) and the Department of Energy (DE-SC0006863). Additional support from the Gaussian Inc., and the University of Washington Student Technology Fund and Royalty Research Fund is gratefully acknowledged. X.L. and C.M.A. are grateful to the Alfred P. Sloan Foundation for Sloan Research Fellowships (20112013). C.M.A. is also grateful to the Camille and Henry Dreyfus Foundation for a Camille Dreyfus Teacher-Scholar Award (2011-2016).

${ }^{1}$ J. R. G. Navarro and M. H. V. Werts, Analyst 138, 583 (2013).

${ }^{2}$ G. K. Joshi, P. J. McClory, B. B. Muhoberac, A. Kumbhar, K. A. Smith, and R. Sardar, J. Phys. Chem. C 116, 20990 (2012).

${ }^{3}$ J. K. Lim, K. Imura, T. Nagahara, S. K. Kim, and H. Okamoto, Chem. Phys. Lett. 412, 41 (2005).

${ }^{4}$ B. S. Guiton, V. Iberi, S. Li, C. M. Leonard, D. N. Parish, P. G. Kotula, M. Varela, G. C. Schatz, S. J. Pennycook, and J. P. Camden, Nano Lett. 11, 3482 (2011).

${ }^{5}$ E. Ringe, B. Sharma, A.-I. Henry, L. D. Marks, and R. P. Van Duyne, Phys. Chem. Chem. Phys. 15, 4110 (2013).

${ }^{6} \mathrm{U}$. Kreibig and M. Vollmer, Optical Properties of Metal Clusters (Springer, Berlin, 1995).

${ }^{7}$ M. A. Mahmoud and M. A. El-Sayed, J. Phys. Chem. Lett. 4, 1541 (2013).

${ }^{8}$ H. Okamoto and K. Imura, J. Phys. Chem. Lett. 4, 2230 (2013).

${ }^{9}$ H. Chen, L. Shao, Q. Li, and J. Wang, Chem. Soc. Rev. 42, 2679 (2013).

${ }^{10}$ L. Polavarapu and L. M. Liz-Marzan, Phys. Chem. Chem. Phys. 15, 5288 (2013).

${ }^{11}$ K. L. Kelly, E. Coronado, L. L. Zhao, and G. C. Schatz, J. Phys. Chem. B 107, 668 (2003).

${ }^{12}$ C. M. Aikens, J. Phys. Chem. Lett. 2, 99 (2011). 
${ }^{13}$ S. M. Morton, D. W. Silverstein, and L. Jensen, Chem. Rev. 111, 3962 (2011).

${ }^{14}$ P. Nordlander and E. Prodan, Nano Lett. 4, 2209 (2004).

${ }^{15}$ P. Nordlander, C. Oubre, E. Prodan, K. Li, and M. I. Stockman, Nano Lett. 4, 899 (2004).

${ }^{16}$ P. K. Jain and M. A. El-Sayed, J. Phys. Chem. C 111, 17451 (2007).

${ }^{17}$ A. J. Haes, C. L. Haynes, A. D. McFarland, G. C. Schatz, R. P. Van Duyne, and S. Zou, MRS Bull. 30, 368 (2005).

${ }^{18}$ K. Kneipp, Y. Wang, H. Kneipp, L. T. Perelman, I. Itzkan, R. R. Dasari, and M. S. Feld, Phys. Rev. Lett. 78, 1667 (1997).

${ }^{19}$ S. Nie and S. R. Emory, Science 275, 1102 (1997).

${ }^{20}$ A. M. Michaels, M. Nirmal, and L. E. Brus, J. Am. Chem. Soc. 121, 9932 (1999).

${ }^{21}$ E. Hutter and J. H. Fendler, Adv. Mater. 16, 1685 (2004).

${ }^{22}$ J. Mullin, N. Valley, M. G. Blaber, and G. C. Schatz, J. Phys. Chem. A 116, 9574 (2012)

${ }^{23}$ K. Saha, S. S. Agasti, C. Kim, X. Li, and V. M. Rotello, Chem. Rev. 112, 2739 (2012)

${ }^{24}$ Z. A. Tagar, Sirajuddin, N. Memon, M. H. Agheem, Y. Junejo, S. S. Hassan, N. H. Kalwar, and M. I. Khattak, Sens. Actuators, B 157, 430 (2011).

${ }^{25}$ S. Jiang, K. Y. Win, S. Liu, C. P. Teng, Y. Zheng, and M.-Y. Han, Nanoscale 5, 3127 (2013).

${ }^{26}$ T. Hirakawa and P. V. Kamat, Langmuir 20, 5645 (2004).

${ }^{27}$ Y.-S. Chen, H. Choi, and P. V. Kamat, J. Am. Chem. Soc. 135, 8822-8825 (2013).

${ }^{28}$ I. H. El-Sayed, X. Huang, and M. A. El-Sayed, Nano Lett. 5, 829 (2005).

${ }^{29}$ X. Huang, I. H. El-Sayed, W. Qian, and M. A. El-Sayed, J. Am. Chem. Soc. 128, 2115 (2006).

${ }^{30}$ Y.-Y. Yu, S.-S. Chang, C.-L. Lee, and C. R. C. Wang, J. Phys. Chem. B 101, 6661 (1997).

${ }^{31}$ S. Link, M. B. Mohamed, and M. A. El-Sayed, J. Phys. Chem. B 103, 3073 (1999).

${ }^{32}$ B. Pietrobon, M. McEachran, and V. Kitaev, ACS Nano 3, 21 (2009).

${ }^{33}$ M.-S. Liao, P. Bonifassi, J. Leszczynski, P. C. Ray, M.-J. Huang, and J. D. Watts, J. Phys. Chem. A 114, 12701 (2010).

${ }^{34}$ H. E. Johnson and C. M. Aikens, J. Phys. Chem. A 113, 4445 (2009).

${ }^{35}$ S. Kümmel, K. Andrae, and P. G. Reinhard, Appl. Phys. B 73, 293 (2001).

${ }^{36}$ C. M. Aikens, S. Li, and G. C. Schatz, J. Phys. Chem. C 112, 11272 (2008).
${ }^{37}$ E. B. Guidez and C. M. Aikens, Nanoscale 4, 4190 (2012).

${ }^{38}$ G.-T. Bae and C. M. Aikens, J. Phys. Chem. C 116, 10356 (2012).

${ }^{39}$ S. Bernadotte, F. Evers, and C. R. Jacob, J. Phys. Chem. C 117, 1863 (2013).

${ }^{40}$ E. B. Guidez and C. M. Aikens, J. Phys. Chem. C 117, 12325 (2013).

${ }^{41}$ M. Walter, J. Akola, O. Lopez-Acevedo, P. D. Jadzinsky, G. Calero, C. J. Ackerson, R. L. Whetten, H. Grönbeck, and H. Häkkinen, Proc. Natl. Acad. Sci. U.S.A. 105, 9157 (2008).

${ }^{42}$ K.-Y. Lian, P. Salek, M. Jin, and D. Ding, J. Chem. Phys. 130, 174701 (2009).

${ }^{43}$ J. Yan, Z. Yuan, and S. Gao, Phys. Rev. Lett. 98, 216602 (2007).

${ }^{44}$ J. Yan and S. Gao, Phys. Rev. B 78, 235413 (2008).

${ }^{45}$ B. Gao, K. Ruud, and Y. Luo, J. Chem. Phys. 137, 194307 (2012).

${ }^{46}$ X. Li, S. M. Smith, A. N. Markevitch, D. A. Romanov, R. J. Levis, and H. B. Schlegel, Phys. Chem. Chem. Phys. 7, 233 (2005).

${ }^{47}$ C. M. Isborn, X. Li, and J. C. Tully, J. Chem. Phys. 126, 134307 (2007).

${ }^{48}$ C. M. Isborn and X. Li, J. Chem. Theor. Comput. 5, 2415 (2009).

${ }^{49}$ M. J. Frisch, G. W. Trucks, H. B. Schlegel, G. E. Scuseria, M. A. Robb, J. R. Cheeseman, G. Scalmani, V. Barone, B. Mennucci, G. A. Petersson, H. Nakatsuji, M. Caricato, X. Li, H. P. Hratchian, A. F. Izmaylov, J. Bloino, G. Zheng, J. L. Sonnenberg, M. Hada, M. Ehara, K. Toyota, R. Fukuda, J. Hasegawa, M. Ishida, T. Nakajima, Y. Honda, O. Kitao, H. Nakai, T. Vreven, J. A. Montgomery Jr., J. E. Peralta, F. Ogliaro, M. Bearpark, J. J. Heyd, E. Brothers, K. N. Kudin, V. N. Staroverov, R. Kobayashi, J. Normand, K. Raghavachari, A. Rendell, J. C. Burant, S. S. Iyengar, J. Tomasi, M. Cossi, N. Rega, J. M. Millam, M. Klene, J. E. Knox, J. B. Cross, V. Bakken, C. Adamo, J. Jaramillo, R. Gomperts, R. E. Stratmann, O. Yazyev, A. J. Austin, R. Cammi, C. Pomelli, J. W. Ochterski, R. L. Martin, K. Morokuma, V. G. Zakrzewski, G. A. Voth, P. Salvador, J. J. Dannenberg, S. Dapprich, A. D. Daniels, Ö. Farkas, J. B. Foresman, J. V. Ortiz, J. Cioslowski, and D. J. Fox, Gaussian 09, Revision H.09+, Gaussian, Inc., Wallingford, CT, 2011

${ }^{50}$ P. J. Hay and W. R. Wadt, J. Chem. Phys. 82, 270 (1985).

${ }^{51}$ P. J. Hay and W. R. Wadt, J. Chem. Phys. 82, 299 (1985).

${ }^{52}$ W. R. Wadt and P. J. Hay, J. Chem. Phys. 82, 284 (1985).

${ }^{53}$ E. B. Guidez and C. M. Aikens, "Plasmon resonance analysis with configuration interaction," Phys. Chem. Chem. Phys. (to be published).

${ }^{54}$ A. E. DePrince III, M. Pelton, J. R. Guest, and S. K. Gray, Phys. Rev. Lett. 107, 196806 (2011). 